\title{
De desastres, políticas y públicos ${ }^{1}$ Disasters, policies and citizens
}

\author{
Patricio Miranda \\ Co-autor: Claudia Atala, Romina Zenteno y Francisco Gatica \\ Patricio Miranda es subdirector de Investigación y Postgrado de la Escuela de Trabajo Social de la Pontificia Universidad Católica \\ de Chile. Magíster en Ética y candidato a doctor en Sociología en la Universidad Alberto Hurtado de Chile. Su dirección electrónica \\ es pmirandr@uc.cl, su dirección postal es Av. Vicuña Mackenna 4860, Macul - Campus San Joaquín. \\ Claudia Atala, Romina Zenteno y Francisco Gatica son estudiantes de quinto año de la Escuela de Trabajo Social UC.
}

\begin{abstract}
Resumen
Se postula que la forma que adquiera la respuesta pública ante desastres públicos dependerá de tres factores críticos: el enfoque paradigmático desde donde se constituye la categoría de desastre (las ciencias naturales, las ciencias de la ingeniería y arquitectónicas o las ciencias sociales); el grado de jerarquización y centralización con que operan las políticas públicas y, fundamentalmente, de una comprensión postconvencional del lugar de los afectados ('los públicos') en todo el ciclo del desastre. Si los esfuerzos de reconstrucción pública pueden reproducir la paradoja observada en modelos convencionales de políticas públicas: ser políticas públicas sin públicos, entonces la eficacia y validez de la respuesta pública ante desastres públicos requiere distanciarse, tanto de lógicas jerárquicas y centralizadoras en las políticas, como de lógicas pre o convencionales en la participación de los afectados. El desarrollo local se piensa como una expresión posible y fructifera en tanto efectivamente 'los públicos' sean co-constructores de la oferta pública ante desastres públicos.
\end{abstract}

Palabras claves. Desastres públicos, politicas públicas, participación, sociedad civil, desarrollo local.

\begin{abstract}
It is proposed that the form public response takes in front to public disasters will depend on three critical factors: the paradigmatic approach from which the category of disaster is built (natural sciences, engineering and architectural sciences or social sciences), the hierarchical and centralized degree in which public policies operate and, crucially, of a postconventional understanding about the place those being affected ('publics') occupy throughout the disaster cycle. If public reconstruction efforts play the paradox observed in conventional models of public policy: public policies without citizens, then the effectiveness and validity of the public response to disasters requires take a distance from both, hierarchical and centralizing logics, as well as from pre or conventional logics about the participation of those affected. Local development is intended as a possible and fruitful expression of involving citizens as co-constructors of the public offer to face public disaster.
\end{abstract}

Keywords. Public disasters, public policies, participation, civil society, local development.

\section{Introducción}

Se postula que la forma que adquiera la respuesta pública ante desastres públicos dependerá de tres factores críticos: el enfoque paradigmático desde donde se constituye la categoría de desastre (las ciencias naturales, las ciencias de la ingeniería y arquitectónicas o las ciencias sociales); el grado de jerarquización y centralización con que operan las políticas públicas y, fundamentalmente, de una comprensión postconvencional de 'los públicos' (los afectados) en todo el ciclo del desastre. Si los esfuerzos de reconstrucción pública pueden reproducir la paradoja observada en modelos convencionales de políticas públicas: ser políticas públicas sin públicos (Aguilar, 2006), entonces la eficacia y validez de la respuesta pública ante desastres públicos requiere distanciarse, tanto de lógicas jerárquicas y centralizadoras en las políticas, como

1 Artículo asociado al proyecto Fondecyt, Normatividad y complejidad en las Políticas Públicas. Un estudio del caso chileno, $N^{o} 1.095 .186$. 
de lógicas pre-convencionales o convencionales en la participación de los afectados. El desarrollo local se piensa como una expresión posible y fructífera en donde 'los públicos' son co-constructores de la oferta pública ante desastres públicos. Ello adquiere mayor sentido si se considera que las limitaciones de la participación ciudadana en las diversas fases de los desastres públicos ha estado estrechamente vinculada con la precaria autonomía de la sociedad civil respecto a los poderes públicos que en sus lógicas jerárquicas y centralizadas se reservan las decisiones fundamentales sin generar mecanismos de participación de los afectados sobre asuntos que tienen que ver con sus propias trayectorias vitales en sus contextos locales (Rodríguez, 1996).

\section{En torno de la categoría de desastre}

La discusión disciplinar respecto de los desastres ha estado marcada por los diversos enfoques dominantes en la investigación sobre ellos (García \& García, 2006). Es así que las formas de ver y tratar los desastres han estado sobredeterminadas por los paradigmas de base dominantes. De alguna manera, la discusión en torno de la conceptualización en intervención en desastres refleja en un campo específico la diversidad irreductible hasta el presente, de enfoques sobre los cuales se han configurado las ciencias sociales. García y García, como resultado de su revisión del estado del arte en torno de la investigación sobre desastres, concluyen que se pueden diferenciar tres enfoques en función de la disciplina dominante: el enfoque físico-naturalista, derivado de las ciencias naturales; el enfoque estructural, derivado de las ciencias aplicadas (ingeniería y arquitectura) y el enfoque social, derivado de las ciencias sociales (2006: 125).

Desde el enfoque físico-naturalista, la mirada se dirige a las causas naturales de fenómenos pre-comprendidos como naturales: terremotos, inundaciones, tsunamis, huracanes, etc. En la medida que en este enfoque hay una sola agencia (la de la naturaleza) y en que se concibe a los desastres como eventos temporales y territorialmente segregados, en los cuales la causa principal se adjudica a procesos físico naturales (Hewitt en García y García, 2006), no queda espacio para la consideración de factores sociales, tanto menos para la consideración de la perspectiva de los sujetos afectados. En planes de reconstrucción puestos bajo este enfoque los públicos' sólo pueden aparecer como uno más de los componentes de la naturaleza siniestrada.

Derivado de las Ciencias de la Ingeniería y Arquitec- tónicas, el Enfoque Estructural "centra la atención en las propiedades físicas del sistema, susceptible de sufrir daños por la acción de un fenómeno externo, en términos de elasticidad, fragilidad y ductilidad de los materiales involucrados" (García \& García, 2005: 126-127). A partir de este enfoque, el desastre surge únicamente desde la amenaza, lo que coincide con el enfoque físico-naturalista, sin embargo incluye un concepto de vulnerabilidad estructural, en la medida en que se centra en métodos probabilísticos de estimación de daños (García \& García, 2005: 127) de carácter físico y económico -cuantificación de bienes perdidos-, además de personas afectadas y pérdidas humanas (García \& García, 2005: 130135). En este sentido, el enfoque realiza un aporte al ampliar el tema a un ámbito multidisciplinario (por ejemplo arquitectos y planificadores, además de sismólogos) dada la consideración de "la amenaza y la vulnerabilidad como variables fundamentales para la planificación física y las normas de construcción de viviendas e infraestructura" (Cardona, 2003: 51, Cit. García \& García, 2005: 127). Desde el enfoque estructural, dominado por las ciencias de la ingeniería y de la arquitectura,

Para que la pregunta por 'los públicos' pueda aparecer en propiedad en las intervenciones en desastres públicos se requiere de un enfoque propiamente social que permita comprender los desastres conjuntamente como producto y "como resultado de procesos sociales, histórica y territorialmente circunscritos y conformados" (Lavell, cit. en García y García, 2006). El estudio de los desastres desde la perspectiva de las ciencias sociales se desarrolló inicialmente en los Estados Unidos teniendo como objeto de investigación las organizaciones complejas en una comunidad que es sacudida por una crisis que excede los recursos de una comunidad (Aguirre, 2004). La ocurrencia de un desastre, además de las incidencias netamente naturales, afecta el orden social y estructural, interfiriendo en las pautas de conducta cotidianas, en donde diversos actores adquieren un rol diferente al establecido. Así, no es puramente la amenaza natural la que produce el desastre, sino el factor social contextual del desastre. Así por ejemplo, un terremoto en una amplia zona desértica no interfiere los flujos de la vida cotidiana de nadie, pero si el mismo evento acontece en un sector poblado podrá llegar a desbordar las capacidades instaladas de manera tal que "aún cuando se trate de amenazas propiamente naturales, en su concreción como eventos destructores factuales [contará] siempre una mediación humana" (Capos, 2003: 2). Agréguese a ello que la existencia de un 
fenómeno natural o socionatural no determina necesariamente un desastre, ni tampoco su magnitud, debido a que "un desastre representa la materialización de condiciones de riesgo existente" (Cardona, 2008:3) en una sociedad. En este sentido, un terremoto o tsunami, no es un desastre por sí solo; para que éste se produzca necesariamente estos deben impactar "en un territorio caracterizado por una estructura social vulnerable a sus impactos y donde la diferenciación interna de la sociedad influye en forma importante en los daños sufridos y en los grupos sociales que sean afectados" (Lavell,1993: 78-79).

Por otra parte, es por lo que hay de social en los desastres que tales no conducen a la misma tragedia, la que en su magnitud dependerá en gran porcentaje del contexto en el cual ocurre. En este sentido, para que haya desastre, es necesario que un fenómeno natural se presente y que "la perturbación generadora del mismo tenga la capacidad de trastocar el funcionamiento del sistema en su totalidad o en alguna de sus provincias o subsistemas, de modo que deje sin efecto temporalmente, la cohesión de los elementos del sistema" (Cardona, 2008: 3). En una palabra, hay desastre público ('social') cuando la capacidad de respuesta de un sistema es rebasada (OMS, cit. Cespédes, 2007). O dicho de otra manera, cuando se produce una ruptura del mundo cotidiano, de lo dado por supuesto, de los cimientos institucionales, normativos y simbólicos sobre los cuales se desarrolla un concreto mundo de la vida (Fouce y Sánchez: 2002). Aquí los tipos y grados de vulnerabilidad que caractericen en un momento dado a una determinada comunidad: vulnerabilidad física o localizacional, económica, social, política, técnica, ideológica, institucional, cultural, educativa, natural y ecológica, dirán del tipo y grado de afectación de los mundos vitales. La magnitud del desastre público resulta así proporcional a la capacidad de resistir, amortiguar, evitar y recuperarse de una comunidad (Wilches-Chaux, 1993 cit. Dehays, 2002); en suma a la capacidad de bloqueo o incapacidad de los habitantes de un territorio "para responder adecuadamente ante la presencia de un riego determinado" (Alonso,2001-2002:15).

Diversos factores sociales (Dettmer,2002:51) concurren en la magnitud final de un desastre; entro otros: el incremento de la densidad de poblaciones asentadas en terrenos anegadizos, áreas costeras vulnerables o cerca de fallas geológicas (Navarro,2006:51), la fragilidad de las economías familiares y colectivas, la ausencia de servicios básicos, la ausencia de oportunidades de educación (Cardona,2003:7, cit. García y García,2005:128), las desigualdades al interior de los países, el acceso restringido a seguros, las disparidades en infraestructura (PNUD,2007-1008:80-82). En este sentido, los desastres, pasan a constituirse como "la resultante de un proceso de generación de vulnerabilidad que tiene su origen en la modificación (intensificación) de pautas económicas y sociales cada vez menos equitativas" (PNUD, 2001, cit. Alonso, 2001-1002:13).

De este modo, desde un enfoque social un desastre público no acontece al margen de las configuraciones históricas de un ordenamiento social dado en un territorio específico (Aguirre, 2004). Configuraciones sedimentadas en largos procesos intergeneracionales, herencia de 'actores muertos', que interpela a los 'actores vivos' a generar redes de articulaciones en pos de la atarea de re-construcción o mejor acaso de re-estructuración.

\section{Políticas públicas para desastres públicos}

Ante desastres públicos la lógica habitual de relación estado-sociedad civil a través de las políticas pública determinará el lugar de los públicos en los esfuerzos de reconstrucción pública. Así, una lógica de políticas centralmente planificadas conducirá a planes de reconstrucción centralmente planificados, colocando a los públicos del desastre en la posición básica de peticionarios y beneficiarios (Salazar y Pinto, 1999); en ningún caso en la condición de co-constructores del esfuerzo público de reconstrucción post-desastre.

En el desarrollo político chileno y latinoamericano la Administración del Estado y el desarrollo de políticas públicas desde ahí no sólo ha administrado, sino que en variados aspectos se ha convertido en el eje articulador de la ciudadanía, en el mecanismo básico que ha permitido y promovido la integración social (Góngora, 1986). Es decir, la formación de políticas en Chile desde distintos puntos de vista, ha sido altamente centralista en su concepción del poder, en su observación del Estado y en la evaluación de la responsabilidad que le cabe a éste en la satisfacción de las demandas de la población. C. Huneeus formula este tópico en cifras: "Los chilenos están consensuados respecto a que el Estado debe tener un rol activo en mejorar la calidad de la salud y la situación de los jubilados, ambas políticas reciben un 98\% de aprobación. Opinan que el Estado debe ofrecer empleo y defender a los consumidores, un 93\% en cada una de estas políticas; debe controlar las diferencias de ingreso entre ricos y pobres: 
$92 \%$, y hasta controlar los precios, un $85 \%$, a pesar de que la inflación sea bajísima. El porcentaje que recibe el menor apoyo es promover el desarrollo de las empresas, $82 \%$, muy alto a la luz de las malas opiniones que tienen los chilenos de los empresarios" (Huneeus, 2003).

La centralidad de las políticas, por tanto, no sólo se refiere a si ellas cumplen función de regulación en todas las dimensiones que ello implica. Las expectativas de la población frente a ellas van más allá: se espera que las políticas logren la integración de la sociedad en dimensiones como las nombradas, una integración que logre conformar un nosotros en el cual cada chileno pueda reconocerse como tal. En tal sentido, la política y sus policies son vistas como la representación de la unidad de la sociedad total, y bajo tal demanda, se le exige que cada diferencia, que cada expectativa sea incorporada como parte de esa unidad. El último Informe de Desarrollo Humano en Chile, lo expone con suficiente dramatismo del siguiente modo: "La convocatoria del Presidente de la República a impulsar un proyecto de país en la perspectiva del Bicentenario 2010 es un doble llamado a todos los chilenos. Convoca a todos porque, como se dijo, el país sólo goza de una diversidad creativa cuando el Nosotros incluye a los Otros. Pero es, además, una convocatoria a todos los ciudadanos en el sentido de que un proyecto de país nada tiene que ver con un 'modelo' único y excluyente. Un proyecto de país no puede ser sino una empresa colectiva a la cual contribuyen el Estado y la sociedad civil, la empresa privada y las organizaciones sociales, las asociaciones gremiales y el mundo académico. Hoy en día, un proyecto de país ha de pensarse como una red -mejor, una red de redes- y, por lo tanto, como una obra plural en todos los colores" (PNUD, 2002).

Si a este desafío cultural de la sociedad chilena se le agrega la 'mentalidad estatista' de la que habla Huneeus, lo que obtenemos por resultado es que la construcción del nosotros, es decir, la construcción de la unidad de la sociedad es, desde la perspectiva de la ciudadanía, una tarea que el mismo Estado debe acometer. El nosotros se refleja en el proyecto país, y el proyecto país es, en última instancia, una selección de la política que debe ser operacionalizada a través de sus políticas públicas. De ello se deriva que las políticas son responsables de la mantención y el fomento de la integración de la sociedad nacional, desde las dimensiones más fácticas de esa exigencia (el cuidado de las fronteras) hasta las más simbólicas (la definición de los valores que caracterizarían al país), pasando, por cierto, por lo que más interesa a esta investigación: la regulación estructural y normativa que generan las policies en materias como trabajo, educación, salud, vivienda.

Bajo estas premisas, la pregunta por la integración social en Chile es respondida tradicionalmente con una inclinación hacia alternativas controladas desde el Estado por la vía de las políticas. Principalmente Claudio Véliz ha investigado para América Latina y Chile el problema del centralismo, el que define bajo las siguientes características (Véliz, 1980):

El centralismo está íntimamente relacionado con la burocracia en el ámbito político;

Se caracteriza por rasgos preindustriales;

Puede ser entendido bajo el concepto weberiano de dominación racional;

Es legalista y enfáticamente civil;

No es una ideología, aunque es posible que se desarrolle una de él, si lo que las diversas formas de centralismo tienen en común se sistematizan.

El centralismo del siglo XX, en tanto, adquiere cuatro dimensiones: la nacional-popular, la desarrollista, la autoritaria y la democrática. No se trata de etapas sucesivas en el desarrollo político chileno, sino de la coexistencia de diversos regímenes que en Chile han parecido estructurarse más bien en términos de etapas (Arellano, 1988). Si bien en un nivel de análisis particular de cada uno de estos regímenes en ellos son sin duda más radicales las diferencias que las semejanzas, la centralidad de la Administración y sus policies frente a los imperativos de regulación estructural y normativa, son una constante en estas cuatro dimensiones, lo que permite confirmar la hipótesis inicial de esta sección relativa a la constitución de lo público a través del Estado.

Este modelo de control estatal jerárquico por medio de policies no sería problemático si la sociedad chilena no estuviera sometida a crecientes presiones de diferenciación estructural y pluralismo normativo expresadas en demandas de inclusión y reconocimiento en el contexto de sistemas funcionales, organizaciones y actores colectivos. La sociedad chilena se complejiza (PNUD, 1986; Lechner, 1991, 1997; Mascareño, 2000, 2005). Por complejización debe entenderse el aumento, densificación e interconexión de las relaciones entre actores, organizaciones y sistemas en el contexto contemporáneo, que conducen a la diversificación de las pretensiones normativas de las diversas esferas diferenciadas en tanto ellas, por su creciente autonomía, ya no responden a un control social de tipo universal y generalizado como el ejercido por el Estado durante 
toda la modernidad y en Chile desde la formación de la República. En un contexto social de creciente complejidad como el de la sociedad chilena contemporánea hay un incremento incontrolado de expectativas normativas en tanto diversos actores u organizaciones van ganando autonomía al desligarse de controles morales tradicionales como los vínculos religiosos o comunitarios, o los lazos de clientelismo político basados en favores recíprocos. Una explosión de nuevas costumbres, hábitos, exigencias morales, estilos de vida marcan los fundamentos de la construcción de nuevas expectativas normativas en la sociedad chilena actual. Como lo señala el PNUD: "Las identidades de clase, religiosas o políticas, aquellas que a mediados del siglo XX permitían a los individuos definir el contenido central de su proyecto vital, han pasado a ser elementos más bien secundarios. $\mathrm{Y}$ ningún otro referente parece ocupar hoy su lugar. Dentro de sus posibilidades reales, ahora las personas están obligadas a elegir sus proyectos vitales. Pero más importante aún y eso es lo propio de la nueva situación, están obligadas a componer esos proyectos a partir de los cambiantes retazos que la sociedad pone a su disposición. Incluso en muchos la velocidad de ampliación de las experiencias posibles hace que la sociedad no alcance siquiera a elaborar orientaciones relativamente satisfactorias para enfrentarlas." (PNUD, 2002: 190-91).

Es decir, no sólo están en proceso de disolución las referencias clásicas para la construcción de identidad, es decir, para la formulación de expectativas normativas de vida, como eran la clase, la religión o la política, sino que además la propia sociedad no decanta nuevos referentes para los problemas que ella misma crea. Por esto, las personas desarrollan estrategias más o menos privadas para intentar construir un campo social, objetivo y temporal apropiado para el despliegue de las nuevas expectativas normativas que construyen.

De estos antecedentes se deriva una fuerte tensión en la construcción de políticas públicas en Chile: el Estado se autocomprende y, por tanto, comprende sus políticas como el centro regulador de las operaciones estructurales de la sociedad y la fuente primaria de un nosotros normativo que debe integrarla éticamente, mientras que en el entorno del Estado y las políticas la diferenciación de la sociedad a nivel de sistemas, organizaciones y actores colectivos presionan por el reconocimiento de su autonomía operativa y sus opciones y expectativas normativas. Es decir, las políticas operan predominantemente en una lógica de control social, mientras que la socie- dad exige descentramiento y coordinación social. El camino regulador fordista, caracterizada por la gestión keynesiana de lo social y por estrategias económicas de escala nacional (Klein, 2005) refuerza, a su modo, las lógicas centralizadoras de larga data en América Latina. Así, el fordismo por una parte y el desarrollo regional por otra, ambos coherentes en sus estrategias, representan el triunfo de la globalidad nacional por sobre la especificidad local, produciéndose la uniformización social, política y económica del territorio nacional (Klein 2005, Jalomç 2009). "Es el triunfo de la sociedad nacional sobre la especificidad local" (Klein, 2005) o, en otras palabras, de la rigidización de una lógica jerárquica en la relación Estado-sociedad civil correspondiente al predominio de una forma de diferenciación estamental (Luhmann, 2007; Mascareño, 2010). Lógica jerárquica que coloca a los 'públicos' de las políticas públicas en condición de beneficiarios y peticionarios pero no co-partícipes en la producción de decisiones vinculantes que les afectan directamente.

\section{Hacia un reconocimiento postconvencional de los 'públicos' en desastres públicos}

Siguiendo la reformulación Habermasiana de los estadios de desarrollo moral de Kohlberg (pre-convencional, convencional y post-convencional), una comprensión post-convencional de los públicos se reconoce en la existencia (o no) de procedimientos de deliberación de los ciudadanos sobre asuntos públicos que les afectan; en un tipo de relación orientada por la búsqueda cooperativa de consensos entre todos los afectados y en el ajuste del comportamiento de los diversos actores a lógicas procedimentales para la fundamentación de normas y decisiones vinculantes (Habermas, 2008). De este modo 'los públicos', pensados en lógica post-convencional, no son meros 'usuarios', 'beneficiarios' o 'clientes' sino sujetos capaces de lenguaje y acción, es decir, interlocutores válidos (Habermas, 2008).

¿Qué implicancias tendría para los esfuerzos de reconstrucción de desastres públicos una consideración post-convencional de los públicos? Un indicador ético de la operación de esta lógica postconvencional sería el grado de participación de los afectados en todo el proceso de formulación, implementación y evaluación del plan de reconstrucción.

En la formulación de los planes de reconstrucción post-desastres públicos, una consideración 
postconvencional de 'los públicos' pasa por que los afectados participen en los procesos de deliberación que conducen a la toma de decisiones vinculantes. Ello implica ir más allá de los actores del sistema político tradicional, incluyendo a potenciales 'beneficiarios', gobierno local, organizaciones de base, gremios, empresas, entre otros. Se trata de una efectiva articulación entre Estado y sociedad civil que recoja y procese las demandas ciudadanas de cara a un desastre público que por definición desborda la agencia de cada actor.

En la implementación de las estrategias de reconstrucción post-desastres públicos, una lógica postconvencional requiere aquí una participación activa de diversos actores, especialmente de los usuarios directos e indirectos de la política, así como también actores que de una u otra manera influyen en la implementación, como gobierno local, organizaciones sociales funcionales $\mathrm{u}$ organizaciones territoriales, las que verán afectada su gestión por los programas o políticas que se ejecuten en los territorios donde operan instituciones gubernamentales o de la sociedad civil.

En cuanto a la evaluación, si ella dice relación con observar los cambios atribuibles a la propuesta de reconstrucción post-desastre público y su relación con los resultados esperados, entonces ésta deberá incluir a actores diversos que en tanto participantes y observadores puedan monitorear los indicadores de logro definidos, con el fin de reinsumar el proceso introduciendo las modificaciones que resulten pertinentes para el logro de los fines estratégicos del proceso. En tal sentido, la antítesis de una lógica postconvencional en la consideración de los públicos sería aquella en que son las personas o grupos que están dentro o forman parte del gobierno quienes definen cuáles son los temas de la agenda y las alternativas de las propuestas de reconstrucción post-catástrofe pública, excluyendo a quienes están fuera o no forman parte del aparato gubernamental (grupos de interés, investigadores, académicos, consultores, medios, opinión pública)

Una lógica post-convencional en políticas, públicos y desastres, exige repensar, entre otras, la relación Estado-sociedad civil. En el contexto de desastres, las intervenciones realizadas por el Estado que han estado articuladas con la activa participación de esa red de asociaciones voluntarias y de organización horizontal de la sociedad que constituye la sociedad civil, han dado muestras de mayor efectividad que aquellas de carácter centralmente planificado. La sociedad civil constituye una plataforma de generación de confianza y de desarrollo de capital social
(Morris y Rodríguez, 2005; Newton, 2001; Saegert, Thompson, y Warren, 2001; en Wong y Leung 2008), de intermediación entre las instituciones del estado y las víctimas del desastre y de catalizador de cuestiones y demandas en la arena pública (Jalali, 2002); todo ello concurre a dar no sólo mayor efectividad (facticidad) a las propuestas de intervención post-desastre sino también, y fundamentalmente, a dar legitimación (validez) a las mismas.

Una de las dinámicas que se levantan contra la centralización y que puede operar con una lógica post-convencional en la consideración de 'los públicos', es el desarrollo local. Pero no entendido desde una lógica neoliberal para la cual este viene a fungir como instancia para deshacer ciertas responsabilidades correspondientes al Estado, que tienen un costo financiero importante, traspasándolas a la sociedad civil (Amin, 2005). En este sentido, el desarrollo local no puede ser visto como el mero hecho de establecer estrategias para el desarrollo en base a divisiones político-administrativas, como la conformación de municipios que responden a un sector geo-delimitado: lo local no está determinado de antemano por estas subdivisiones, sino más bien por un cierto número de condiciones expresados en el nivel socioeconómico, y el cultural (Arocena, 2002). La sola descentralización parece responder más bien a una lógica funcional del modelo neoliberal, y no a una visión de lo público signada por la lógica de democratización de la sociedad (Gallicchio, 2004)

Una lógica post-convencional en el desarrollo local reclama un cambio en la visión del Estado con el fin de configurar nuevos espacios de autonomía donde puedan generarse nuevas solidaridades locales, formas activas de identidad que les reconozcan a las comunidades locales un mayor poder de participación en la toma de decisiones (Teisserenc, 1994; Tremblay y Fontan, 1997; en Klein, 2005). Lo común que se puede extraer de estas visiones corresponde a que el Estado ya no es el único actor en materia de desarrollo (Klein, 2005). El redescubrimiento de lo local en el caso de América Latina coincide con la crisis estructural de la configuración de un estado centralizador y benefactor (Jalomo, 2009). Frente a una lógica de desarrollo inducido jerárquicamente por el estado emergen fórmulas alternativas de desarrollo como el 'autodesarrollo', el 'desarrollo endógeno', el 'desarrollo autocentrado', el 'desarrollo integrado' y el 'desarrollo a escala humana', entre otros (Arocena 1995; Klein 2005; Jalomo 2009). El desarrollo local como factor de democratización y desarrollo sustentable emerge 
como una alternativa al desarrollo regional y nacional predominante (Gallicchio, 2004).

La fuerte centralización como fenómeno socio-organizativo característico del modelo fordista produjo en América Latina un debilitamiento y una desvalorización de lo local (Arocena, 2002). El triunfo del centralismo ha ido de la mano de la devaluación de las iniciativas locales, de una actitud de espera de las soluciones que vendrán del 'centro' para calmar las necesidades más elementales. El predominio de las tendencias históricas centralistas ha tenido entonces como efecto la conformación de un régimen local débil, con dificultades para constituirse en instancia fundamental del funcionamiento democrático (Arocena, 2002). En la lógica dominante del sistema político-administrativo centralizado que corresponde al tipo sectorial-vertical, el sistema se estructura en base a sectores especializados en las distintas áreas de acción: administración central formada por ministerios, que funcionan bajo cierto tipo de racionalidad a lo largo del territorio nacional, extendiendo una acción uniforme. Así, el poder central obtiene el dominio absoluto. Cada uno de estos ministerios se especializa en un área determinada, generándose un monopolio, no admitiendo ninguna iniciativa generada desde fuera de su ámbito centralizado de decisión.

Si el trabajo desde lo local es la única manera de concebir lo universal (Arocena, 2002), entonces en una visión integradora, ascendente, que combina los elementos socioeconómicos y cultural identitarios (Arocena, 2002) lo local adquiere una gran importancia en su interrelación con estrategias globales. El desarrollo desde la base permite construir desde la diferencia, establecer tejidos complejos regidos por mecanismos de negociación, integrar lugar de trabajo y lugar de vida, reconocer las especificidades de los recursos naturales (Arocena, 2002). En la escena local converge la necesidad de crear riqueza con la necesidad de salvaguardar los recursos naturales, la urgencia por generar empleos con la urgencia por responder a las necesidades esenciales de la población: en la escena local, se expresa como en ningún otro nivel, la articulación entre lo singular y lo universal (Arocena, 2002). Ello en la medida en que se pueda sostener que la "mejor manera de resolver los problemas comunes es, sin duda, en comunidad" (Salazar y Pinto, 1999). Si los esfuerzos públicos de reconstrucción post-desastre apuntan a "resolver los problemas de un colectivo social determinado (articulando sus necesidades, recursos, saberes y pareceres, en la perspectiva de realizar una acción conjunta), entonces el único modo co- herente de hacer [reconstrucción] es involucrando a toda la comunidad, tanto en la fase de diagnóstico (asamblea), toma de decisión (acuerdo), como en la ejecución (acción)" (Salazar y Pinto, 1999).

En una perspectiva de desarrollo local no subsumida en lógicas neoliberales o meramente funcionales, una comprensión post-convencional de los públicos en contextos de desastres no sólo potencia la sociedad civil sino que permite mejor enfrentar los factores críticos que la literatura especializada ha identificado como prioritarios, entre ellos, la desorganización social, la presencia de conflictos, la destrucción de las redes formales e informales. Los esfuerzos de reconstrucción se potencian cuando todos los miembros de las comunidades locales afectadas en un territorio se articulan en respuesta a los desafíos externos a ellas (Adeola, 2001; fritz, 1961; Sweet, 1998; en Yoon 2006).

\section{Reflexiones finales}

Los resultados preliminares de la investigación Fondecyt: Normatividad y complejidad en las Políticas Públicas. Un estudio del caso chileno ( $\left.{ }^{\circ} 1095186\right)$, llevada adelante actualmente por el autor principal en torno de dos factores críticos que dan forma a las políticas: la visión de sociedad y el referencial normativo implícito en ellas, indican que, tanto en modelos convencionales de análisis de políticas, como en las políticas en Chile en la última década, priman visiones jerárquicas de sociedad junto a criterios normativos fundamentalmente de tipo pre-convencional o convencional, en la terminología kohlbergiano-habermasiana; es decir, formas de decisión moral asociadas a intereses particularistas o mecánicamente legalistas, que escapan a la aplicación contextualizada de principios morales de alcance post-convencional.

La investigación referida mostró que en modelos convencionales de análisis de políticas públicas (racionalista, incrementalista, de elección pública, institucionalista, de grupos y de elites) predominan -parafraseando a Regonini (1991)- dos figuras de policy makers: la del 'actor omnisciente' y la del 'tutor moral' de la sociedad (Jobert, 2004). La primera, reflejo de una visión jerárquica o monocéntrica de sociedad, asociada a una forma de diferenciación social estratificada (Luhmann, 2007); la segunda, asociada a un tipo de normatividad particularista correspondiente a un estadio de moralidad de tipo convencional (Habermas, 1985). La prevalencia de ambas figuras dice de una relación de homología entre formas de diferenciación social y formas de 
diferenciación normativa (Miranda, 2010). Los resultados preliminares de la fase empírica de la investigación centrada en policy makers y policy managers en Chile, resultan consistentes con lo observado en los modelos referidos ${ }^{2}$.

Ante las transformaciones estructurales y semánticas en curso en la sociedad chilena se torna un imperativo ético el discutir las modalidades, alcances, limitaciones y perspectivas de la participación ciudadana en la prevención de e intervención en desastres; participación imprescindible de una sociedad civil que hace parte de la configuración de la esfera pública en pos de formas de des-centralización de las decisiones fundamentales en las que se juegan las condiciones existenciales vitales de las comunidades locales (Rodríguez, 1996). El señalado centralismo que funge en las lógicas y estrategias pre y post-desastre, reproduce las formas verticales del proceso decisional dominante en las políticas públicas, 'asignando' al nivel local y sus actores un papel reducido principalmente a la ejecución de las acciones diseñadas por el nivel central (Rodríguez, D. 1996). Todo ello paradojalmente dice, en suma, de políticas públicas ante desastres públicos, sin públicos.

\section{Bibliografía}

ALONSO, I. (2001-2002). Tercer mundo, desarrollo, desastres y tecnología. Una mirada desde la geografía. Serie geográfica, Tecnologías geográficas para el desarrollo: Experiencias de aplicación en América Latina, No 10, 11 26. Recuperadoel8 de Abril2010, dehttp://www.geogra. uah.es/inicio/revista/pdfrevista10/OK-01-Revista-Articulo-Ignacio\%20Alonso\%20-11\%20a\%2026.pdf

AROCENA, JOSÉ. (1998). "Discutiendo lo local, coordenadas del debate" Cuadernos del CLAEH, No 41, Montevideo.

AROCENA, JOSÉ. (2002). El desarrollo local: un desafío contemporáneo. Taururs, Universidad Católica. Segunda Edición. Uruguay.

CAMPOS, A. (2003). Educación y Prevención de Desastres. UNICEF, FLACSO, LA RED (Red de Estudios Sociales en Prevención de Desastres en América Latina) [Versión Electrónica]. Recuperado el 9 de Junio de 2010, de http://www.desenredando.org/public/ libros/2000/eypd/EducacionYPrevencionDeDesastres1.0.1.pdf
CARDONA, O. (2008). Medición de la gestión del riesgo en América Latina. Revista Internacional de Sostenibilidad, Tecnología y Humanismo. №3, 1-20. Recuperado el 13 de Abril de 2010 de http://upcommons.upc.edu/ revistes/bitstream/2099/7056/1/cardona.pdf

CARPIO MARTÍN, JOSÉ. (2000). Desarrollo local para un nuevo desarrollo rural. Anales de Geografía de la Universidad Complutense. Madrid.

CÉSPEDES M. \& VIVIAN, M. (2007). Los desastres, la información y el Centro Latinoamericano de Medicina de Desastres. ACIMED [versión electrónica], ISSN 1024-9435, vol.16, No2. Recuperado el 10 de Junio de 2010, de http://scielo.sld.cu/scielo. php?script=sci_arttext\&pid=S1024-94352007000800 006\&lng=es\&nrm=iso>

DEHAYS, J. (2002, Junio). Fenómenos Naturales, Concentración Urbana y Desastres en América Latina. Perfiles Latinoamericanos: Revista de la Facultad Latinoamericana de Ciencias Sociales, ISSN 0188-7653, No. 20, 177-206. Recuperado el 14 de Abril de 2010, de http:// dialnet.unirioja.es/servlet/articulo?codigo $=2211535$

DETTMER, J. (2002). Educación y desastres, reflexiones sobre el caso de México. Revista Latinoamericana de Estudios Educativos, $2^{\circ}$ trimestre, vol.32, No002, 43-74. Recuperado el 7 de Abril de 2010, de http://redalyc.uaemex.mx/redalyc/pdf/270/27032203.pdf

ESCOBAR, A. (2004). Participación Ciudadana y Políticas Públicas: Una problematización acerca de la relación Estado y Sociedad Civil en América Latina en la última década. Rev. austral cienc. soc., 2004, №. 8 , pp. 97-108. ISSN 0718-1795

FOUCE, GUILLERMO y SÁNCHEZ, ESTEBAN (2002). Intervención psicosociológica en situaciones de estrés agudo: consideraciones teóricas y sugerencias para la intervención en situaciones de emergencia. Anuario de Psicologá, Facultad de psicología. Universidad Complutense de Madrid.

GALLICCHIO, ENRIQUE. (2004). El desarrollo local en América Latina. Estrategia política basada en la construcción de capital social. Programa de desarrollo local. CLAEH, Uruguay.

GARCÍA \& GARCÍA. (2005). Concepciones paradigmáticas subyacentes en el manejo e investigación sobre desastres. Revista de Investigación (Instituto Pedagógico de Caracas), ISSN 1010-2914, № 57, 123-142. Recuperado el 13 de Abril de 2010, de http://dialnet. unirioja.es/servlet/articulo?codigo=2053503

2 Varios temas y consecuencias de esto están siendo y serán abordadas en el nuevo proyecto presentado al Fondecyt Regular 2011, entre otros, el que levanta el desafío de pensar políticas y modelos de análisis de políticas que en su visión acerca del funcionamiento de la sociedad (Stein y Tommasi, 2006), reflejen simultáneamente el carácter crecientemente heterárquico o policéntrico de sociedades modernas y la creciente demanda de una normatividad de tipo postconvencional acorde al aumento de diversidad normativa (Miranda, 2010). 
GÓNGORA, M. (1986). Ensayo histórico sobre la noción de Estado en Chile en los siglos XIX y XX, Editorial Universitaria, Santiago.

HUNEEUS, C. (2003). Chile un país dividido. La actualidad del pasado, Catalonia, Santiago.

JALOMO AGUIRRE, FRANCISCO (2009). Desarrollo local en contextos metropolitanos. Universidad de Guadalajara, Jalisco, México.

KLEIN, JUAN-LUIS. (2005). Iniciativa local y desarrollo: respuesta social a la globalización neoliberal. Revista eure, Vol. XXXI, No 94. Santiago de Chile.

LAVELL, T. (1993). Ciencias Sociales y desastres naturales en América Latina: un encuentro inconcluso. Revista Electrónica Eure, vol.19, No 58, 73-84. Recuperado el 14 de Abril de 2010, de http://www.eure. cl/media/uploads/pdf/Doc0006.pdf

LECHNER, N. (1991). El estado en el contexto de la modernidad, en Lechner, Norbert, René Millán y Francisco Valdés (coords.), Reforma del estado y coordinación social, Plaza y Valdés, México, 1991, pp. 39-54, p. 52.

LECHNER, N. (1997). Tres formas de coordinación social, Revista de la CEPAL, 61, 1997, pp. 7-17.

MASCAREÑO, A. (2000). Diferenciación funcional en América Latina: los contornos de una sociedad concéntrica y los dilemas de su transformación, en Persona y Sociedad, Vol. XIV, Nr. 1, April 2000, pp.187-207.

MASCAREÑO, A. y MEREMINSKAYA, E. (2005). Hacia la consolidación de la equidad: Un defensor del pueblo para Chile, en Colección de Investigaciones Jurídicas $\mathrm{N}^{\circ} 7$, Escuela de Derecho Universidad Alberto Hurtado, Santiago.

NAVARRO, V. (2006). Los desastres en su interacción con la ciencia, la tecnología y la sociedad. Revista Electrónica de las Ciencias Médicas en Cienfuegos, Medisur, vol.4, No2, 50-58. Recuperado el 14 de Mayo de 2010, de http://www.medisur.sld.cu/index.php/medisur/article/view/202/4915

PÉREZ, K. (2005). Gestión de desastres. Diccionario de acción humanitaria y cooperación al desarrollo. Disponible en http://www.dicc.hegoa.ehu.es/listar/ mostrar/228

RODRÍGUEZ, D. (1996). EL FUTURO DE LA PARTICIPACIÓN CIUDADANA ANTE LOS DESASTRES. Desastres y Sociedad, No 6 / Año 4. Disponible en http://www.desenredando.org/public/revistas/dys/ rdys06/dys6-1.0-efpcad.pdf

PNUD. (2002). Desarrollo Humano en Chile. Nosotros los chilenos: un desafío cultural, PNUD, Santiago.

VÉLIZ, C. (1980). The centralist tradition of Latinamerica, Princeton University Press, Princeton, New Jersey.
WONG, HUNG y LEUNG, TERRY. (2008). Collaborative vs. Adversarial. Relationship between the State and civil society in facing public disaster. The case of Hong Kong in the SARS crisis. Asian Pacific Journal of Social Work and Development, Volume 18, No2.

YOON, INTAE. (2006). A Mixed-Method Study of Princeville's Rebuilding from the Flood of 1999: Lessons on the Importance of Invisibile Community Assets. East Carolina University. 